\title{
Evening Avoidance of Large Meals alongside Evening Exercise to Improve Maternal and Child Health
}

\section{Akbar Nikkhah}

Department of Animal Sciences, Faculty of Agricultural Sciences, University of Zanjan, Iran

*Corresponding author: Nikkhah A, Chief Highly Distinguished Professor, Department of Animal Sciences, Faculty of Agricultural Sciences, University of Zanjan, Foremost Principal Highly Distinguished Elite-Generating Scientist, National Elite Foundation, Iran, E-mail: anikkha@yahoo.com

Received date: January 18, 2016; Accepted date: January 28, 2016; Published date: February 27, 2016

Copyright: ( 2016 Nikkhah A. This is an open-access article distributed under the terms of the Creative Commons Attribution License, which permits unrestricted use, distribution, and reproduction in any medium, provided the original author and source are credited.

\begin{abstract}
This perspective article describes a pragmatic methodology to improve mother and child health through reduced evening eating and increased evening exercise during gestation and lactation. Evening avoidance of large meals complemented by evening exercise, when insulin efficiency decreases helps pregnant and lactating mothers better cope with the changing endocrinology.
\end{abstract}

Keywords: Evening; Eating; Exercise; Pregnancy; Child health

\section{Philosophy and Discussion}

Insulin efficiency and glucose tolerance decrease as evening and night begin. This evolutionary trend requires matching eating and exercise programs. Today's busy life has been an excuse for the modern man to have little physical training. This alongside interrupted daily food meals makes the body prone to numerous metabolic abnormalities. This article, thus, innovatively develops a pragmatic lifestyle that involves little evening eating and increased evening physical work to improve endocrinology and health, especially in pregnant and lactating women [1-4].

Pregnancy diabetes is a common metabolic disorder that jeopardizes mother and child health. Pregnant women must be trained to have their major food meals during day and avoid eating much during evening and night times. Night eating may easily exacerbate the conditions that lead to hyperglycaemia and impaired insulin action. The problem gets worse when no or little physical exercise occurs. Evening requires more physical work than morning in enabling the human body to keep harmonies between internal physiology and the environment [5-7].

Since human physiology functions on a circadian nature, each and every meal should ideally have its matching exercise session on a 24-h basis. As human has evolved to have limited metabolism overnight, large evening meals are not suited towards optimal health. However, due to the very busy day-time schedule of the modern man, evenings and nights are often devoted to social activities. This is linked to evening overeating and suboptimal eating patterns that cause obesity and related metabolic disorders. Therefore, it is critical that evening exercise be performed to minimize the above risk of diseases.

In pragmatic words, highly disciplined afternoon and evening exercise must start early enough preferably when pregnancy starts.
Such a prolonged program will facilitate physical work during heavy pregnancy times. Normally, physical work occurs effectively when no eating occurs at least for few hours before exercise starts. However, some limited eating and drinking needs to be done post-exercise to healthily regulate blood pressure and heart performance. In a nutshell, evening exercise is a good complement to evening limit-eating, collectively improving heart, liver, kidney, and total splanchnic health and metabolism.

\section{Acknowledgments}

Thanks to the Ministry of Science Research and Technology, and National Elite Foundation for supporting the author's global initiatives and programs of optimizing science edification in the third millennium.

\section{References}

1. Nikkhah A (2015) Harmonizing eating and exercise circadian rhythms for optimal glucose-insulin and vascular physiology. Int J Diabetol Vasc Dis Res 3: 87-88.

2. Nikkhah A (2015) Secure weight management via fitting circadian patterns of physical activity, resting and eating. Adv Weigh Manag Obes Cont 2: 00023 .

3. Nikkhah A (2015) Circadian fitting of exercise and eating patterns: The secret of healthy life. J Bioprocess Biotech 5: e129.

4. Nikkhah A (2015) Breast health progress through exercise-driven lactation: A pragmatic bioprocess to prevent cancer. J Bioprocess Biotechniq 5: e127.

5. Nikkhah A (2015) Optimizing gestation and early life physiology through timing of energy turnover: Bioprocessing of human life. J Bioprocess Biotechniq 5: e125.

6. Nikkhah A (2015) Avoid large night meals to stay fit. J. Obes. Weight Loss Ther 4: e115.

7. Nikkhah A (2014) When to eat to beat obesity and diabetes? Journal of Diabetes and Metabolism. 